\title{
Advantage of Using 8K Ultra-High-Definition Television System for Kasai Portoenterostomy for Biliary Atresia
}

\author{
Hisayuki Miyagi ${ }^{1}$ Daisuke Ishii ${ }^{1}$ Masatoshi Hirasawa ${ }^{1}$ Tatsuya Shonaka ${ }^{2}$ Yasuo Sumi ${ }^{2}$ \\ Nobuyoshi Azuma ${ }^{3}$
}

${ }^{1}$ Division of Pediatric Surgery, Department of Surgery, Asahikawa Medical University, Asahikawa, Hokkaido, Japan

2 Division of Gastrointestinal Surgery, Department of Surgery, Asahikawa Medical University, Asahikawa, Hokkaido, Japan

${ }^{3}$ Department of Vascular Surgery, Asahikawa Medical University, Asahikawa, Hokkaido, Japan

Eur J Pediatr Surg Rep 2021;9:e5-e8.

\author{
Address for correspondence Hisayuki Miyagi, MD, PhD, Division of \\ Pediatric Surgery, Department of Surgery, Asahikawa Medical \\ University, Asahikawa, Hokkaido 078-8510, Japan \\ (e-mail: miyagi@mvj.biglobe.ne.jp).
}

\begin{abstract}
Keywords

- biliary atresia

- 8K ultra-highdefinition television system

- Kasai portoenterostomy

- Kasai procedure

Kasai portoenterostomy (KPE) is currently the first-line treatment for biliary atresia. Many pediatric surgeons have reported that the dissection of the fibrous remnant at the porta hepatis is one of the most important components of this procedure. Furthermore, laparoscopic portoenterostomy is being increasingly used to treat biliary atresia.

An advantage of laparoscopic surgery is that surgeons can more easily identify microbiliary ducts, owing to the magnification. We report the case of a 61-day-old girl on whom we performed an exploratory laparotomy and diagnosed type III biliary atresia using intraoperative cholangiography. For the first time, we performed an open KPE using an $8 \mathrm{~K}$ ultra-high-definition television system. This allowed us to clearly view the porta hepatis and to successfully perform the portoenterostomy.
\end{abstract}

\section{New Insights and the Importance for the Pediatric Surgeon}

We performed open Kasai portoenterostomy (KPE) using an $8 \mathrm{~K}$ ultra-high-definition laparoscope to facilitate examination of the portal region and confirmed outflow of bile from the resection surface and successfully performed open KPE using this laparoscopic system (8K Kasai procedure). This is the first report to describe the performance of the $8 \mathrm{~K}$ Kasai procedure, which we expect to be useful for both laparoscopic portoenterostomy and open KPE.

\section{Introduction}

The first successful surgical procedure for noncorrectable biliary atresia was reported in 1959 by Kasai et al. ${ }^{1}$ More recently, Davenport et al reported that the 10 -year native liver survival estimate was $40 \%$ in England and Wales, ${ }^{2}$ Chardot et al reported the 10-year actuarial survival with a

received

July 30, 2020

accepted after revision

October 9, 2020
DOI https://doi.org/

10.1055/s-0040-1721466.

ISSN 2194-7619. native liver to be $29 \%$ in France, ${ }^{3}$ and Nio reported the 20 year native liver survival to be $49 \%$ in Japan. ${ }^{4}$ Over the years, many technical modifications have been made to Kasai portoenterostomy (KPE), and many pediatric surgeons have reported that the dissection of the fibrous remnant at the porta hepatis is one of the most important components of this procedure. ${ }^{5}$
(C) 2021. The Author(s).

This is an open access article published by Thieme under the terms of the Creative Commons Attribution License, permitting unrestricted use, distribution, and reproduction so long as the original work is properly cited. (https://creativecommons.org/licenses/by/4.0/)

Georg Thieme Verlag KG, Rüdigerstraße 14, 70469 Stuttgart, Germany 
Laparoscopy is now widely used in pediatric patients, and laparoscopic portoenterostomy (LPE) was first used to treat biliary atresia in $2002 .{ }^{6}$ Some researchers have since reported favorable outcomes, ${ }^{7,8}$ but others have reported that LPE is associated with a lower survival rate than open KPE when applied to native livers. ${ }^{9}$

At our institution, we perform modified open KPE and often encounter patients in whom the bile outflow site cannot be easily observed during the resection of the fibrous remnant. Here, we report a case in which we performed open KPE and were able to confirm bile outflow from the dissection surface using an $8 \mathrm{~K}$ ultra-high-definition (UHD) television system to view the porta hepatis, which allowed us to perform the portoenterostomy successfully.

\section{Case Report}

The patient was a 61-day-old girl. She was born at a gestational age of 37 weeks and had a birth weight of $2.41 \mathrm{~kg}$. She had a slightly high galactose concentration on mass screening for congenital metabolic abnormalities at 4 days of age. She was examined at another hospital at 13 days of age, when she exhibited white feces and a high circulating direct bilirubin concentration. Her gallbladder could not be identified by abdominal ultrasonography; therefore, biliary atresia was suspected. At the age of 39 days, bile excretion was not observed on hepatobiliary scintigraphy, and hepatobiliary scintigraphy at the previous hospital had not confirmed excretion into the intestinal tract. Due to the novel coronavirus pandemic and the circumstances of the family, her referral to our hospital was slightly delayed. The patient was admitted to our department at the age of 57 days and underwent exploratory laparotomy at the age of 61 days, when she was diagnosed with type III biliary atresia (the type of extrahepatic biliary obstruction was determined using the Japanese Society of Pediatric Surgeons Classification $^{1}$ ) using intraoperative cholangiography (-Fig. 1A) and underwent open KPE using the 8K UHD television system.

A right subcostal laparotomy was performed and intraoperative cholangiography was performed via the atrophied gallbladder. Only the gallbladder could be detected, and the contrast agent leaked from the lateral aspect under compression. A diagnosis of type III biliary atresia was made on the basis of the cholangiographic and gross findings, and open KPE was performed.


Fig. 1 (A) Intraoperative cholangiography was performed by inserting a tube from the bottom of the gallbladder (yellow arrow), but neither the common hepatic duct nor the common bile duct could be delineated, and compression resulted in leakage from the lateral aspect of the gallbladder. (B) The view of the surgical field was magnified on a 70-inch monitor and the site of bile outflow was confirmed by several pediatric surgeons (the fingers of the surgeon and outflow site are shown on the monitor). (C) View of the porta hepatis in the standard high-definition mode. Photograph comparing the resolution using 3-0 suture material ( $\sim 0.2 \mathrm{~mm}$ in diameter) in the standard high-definition mode. Bile outflow could not be visually confirmed. (D) View of the porta hepatis in $8 \mathrm{~K}$ ultra-high-definition mode. Outflow of bile from a site near the right hepatic artery was confirmed (yellow arrows). 
The connective tissue in the portal region was dissected using Metzenbaum scissors at the level of the hepatic capsule, from the medial side of the left portal vein toward the medial side of the right portal vein, while applying gauze saturated with warm physiological saline to aid hemostasis.

At that time, the site of dissection was confirmed by examining the portal region using the camera of the $8 \mathrm{~K}$ UHD laparoscopic system, and the biliary outflow site was examined after dissection. Bile outflow near the right hepatic artery was observed in 8K UHD mode (-Figs. 1B, D and 2A), but was not visible in high-definition mode (-Fig. 1C).

The jejunum was separated at a point $30 \mathrm{~cm}$ from the ligament of Treitz, an ascending limb of $40 \mathrm{~cm}$ in length was elevated in front of the colon, and portoenterostomy was performed. The jejunum was anastomosed to the porta hepatis using 5-0 PDS II, which sufficiently covered the biliary outflow site ( - Fig. 2B).

The biliary tract was reconstructed using the Roux-en-Y procedure, with the creation of a Nakajo-type enteric antireflux valve at a point $10 \mathrm{~cm}$ toward the liver from the Rouxen-Y limb and the Roux-en-Y limb was secured at an acute angle by suturing the seromuscular layer, to prevent food reflux. We performed an antireflux plasty using the intus- suscepted valve Roux-en-Y procedure, according to the technique performed at Tohoku University Hospital. ${ }^{2}$

The postoperative course was uneventful. Oral feeding was initiated on postoperative day (POD) 3, and the drain was removed on POD 6. Steroid administration was started, according to the protocol of the Japanese Biliary Atresia Society, on POD 7, and the patient was discharged on POD 17 with a total bilirubin concentration of $2.4 \mathrm{mg} / \mathrm{dL}$. Thereafter, the patient was followed up on an outpatient basis, involving weekly attendance at the clinic during the immediate period following discharge, and once monthly visits when her blood parameters had normalized. She was initially followed up by both a pediatric surgeon and a pediatrician, but in the longer term she is being monitored principally by a pediatric surgeon. At the time of writing (POD 115), no cholangitis has been noted and her total bilirubin concentration is $0.5 \mathrm{mg} / \mathrm{dL}$ ( Fig. 2C).

\section{Discussion}

Many modifications have been made to the KPE technique over the years. In particular, the method used to determine the area and depth of resection of periportal connective
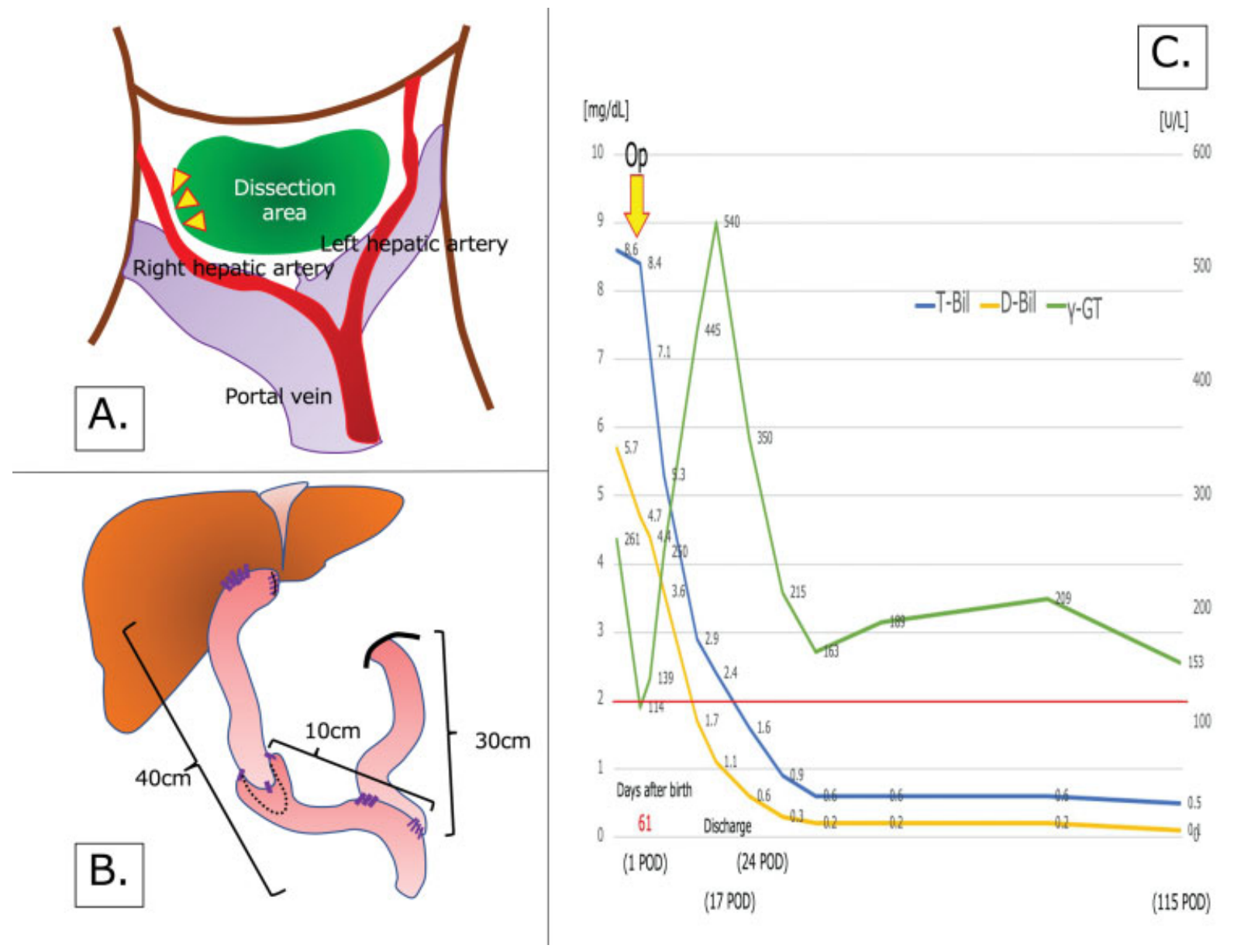

Fig. 2 (A and B) Schema of the surgery. (A) Outflow of bile was confirmed near the entrance of the right hepatic artery. (B) Schema of the biliary tract reconstruction using an artificial enteric valve. At our institution, we separate the jejunum $30 \mathrm{~cm}$ from the ligament of Treitz, such that the limb for a potential liver transplant is $40 \mathrm{~cm}$ long. Then, the limb is elevated to the porta hepatis, through the front of the transverse colon (antecolic Roux limb route). (C) Trends in laboratory data. Graph showing changes in the circulating T-Bil and D-Bil concentrations, and gammaglutamyltransferase activity over time. D-Bil, direct bilirubin; POD, postoperative day; T-Bil, total bilirubin. 
tissue is an important issue. Nio et $\mathrm{al}^{5}$ reported that the level of the hepatic capsule is appropriate as the resection line for the periportal connective tissue, and our department uses the same approach.

Previous studies have shown that the identification of microbiliary channels is often possible by magnifying laparoscopic images. ${ }^{10}$ Laparoscopy may be inferior to microscopy in this respect, but it is not practical to insert and withdraw a microscope during LPE or open KPE. Therefore, we decided to use an 8K UHD laparoscopic system. An $8 \mathrm{~K}$ UHD laparoscope was developed that was based on ultrahigh-resolution 8K UHD imaging technology $(7,680 \times 4,320$ pixels), which should enable physicians to perform safer and more efficient laparoscopic surgery. The 8K UHD laparoscopic camera weighed $>2 \mathrm{~kg}$ in 2014, but its weight was reduced to $370 \mathrm{~g}$ by Kairos Co. Ltd., and its commercial distribution as a medical device was approved in 2017. ${ }^{11}$ Because the $8 \mathrm{~K}$ laparoscope has 16 times higher resolution than a conventional high-vision laparoscope $(1,920 \times 1,080$ pixels), it has become possible to identify fine blood vessels and membrane structures and distinguish important nerves that were previously difficult to identify visually. ${ }^{11}$

We used the 8K UHD laparoscopic system to examine the hepatic portal region of a child with biliary atresia who was undergoing KPE, and observed slight bile outflow. Anastomosis was possible at the intended site, and her postoperative course was uneventful. Therefore, we expect the 8K UHD laparoscopic camera to be useful for laparoscopic and open surgery.

This procedure has two main limitations. First, the $8 \mathrm{~K}$ UHD laparoscopic device contains a heated light source. Therefore, it should not be applied directly to the patient, its duration of use should be minimized, and the application of the light to a single site for a long period of time should be avoided. Second, although bile outflow was confirmed in the present case, it may be difficult to detect, depending on the type and stage of the disease. We do not recommend the use of the $8 \mathrm{~K}$ system in cases in which bile outflow is clearly visible to the naked eye during surgery. However, by preparing an $8 \mathrm{~K}$ UHD system preoperatively, we expect that bile outflow, which was previously invisible to the naked eye, may be rendered visible, and anastomosis can be facilitated. In the unlikely event that the 8K UHD system does not show bile outflow, the anastomotic site can be determined anatomically and empirically. In addition, long-term outcome data have yet to be collected, and therefore further cases should be studied over a longer period of time.

In conclusion, we performed open KPE using an 8K UHD laparoscope to examine the portal region. We confirmed outflow of bile from the resection surface and successfully performed open KPE using the laparoscopic system (8K Kasai procedure). This is the first report to describe the performance of this technique, which we expect to be useful for both LPE and open KPE.

\section{Ethics Approval}

This study was approved by the Asahikawa Medical University Research Ethics Committee (registration num- ber: 20035), and written informed consent was obtained from the parents of the patient for publication of this case report and any accompanying images. A copy of the consent document is available for review by the Editorin-Chief of this journal on request.

\section{Funding}

None.

\section{Conflict of Interest}

None of the authors have any conflict of interest.

\section{Acknowledgments}

We thank Angela Morben, DVM, ELS, Cathel Kerr, BSc, PhD, and Mark Cleasby, PhD, from Edanz Group (https://enauthor-services.edanzgroup.com/ac), for editing drafts of this manuscript. We also thank Etsushi Tsuchida, Toshio Okamoto, and Ken Nagaya, Pediatricians from the Department of Pediatrics, Asahikawa Medical University; Seri M. Maruyama and Sumiko Sako, Anesthesiologists from the Department of Anesthesiology and Critical Care Medicine, Asahikawa Medical University; Katsuaki Naya, Clinical Engineer from the Surgical Center of Asahikawa Medical University Hospital; and Akitoshi Yoshida, President of Asahikawa Medical University, for their assistance.

\section{References}

1 Nio M, Ohi R. Biliary atresia. Semin Pediatr Surg 2000;9(04): 177-186

2 Davenport M, Ong E, Sharif K, et al. Biliary atresia in England and Wales: results of centralization and new benchmark. J Pediatr Surg 2011;46(09):1689-1694

3 Chardot C, Carton M, Spire-Bendelac N, Le Pommelet C, Golmard JL, Auvert B. Prognosis of biliary atresia in the era of liver transplantation: French national study from 1986 to 1996 . Hepatology 1999;30(03):606-611

4 Nio M. Japanese Biliary Atresia Registry. Pediatr Surg Int 2017;33 (12):1319-1325

5 Nio M, Wada M, Sasaki H, Kazama T, Tanaka H, Kudo H. Technical standardization of Kasai portoenterostomy for biliary atresia. J Pediatr Surg 2016;51(12):2105-2108

6 Esteves E, Clemente Neto E, Ottaiano Neto M, Devanir J Jr, Esteves Pereira R. Laparoscopic Kasai portoenterostomy for biliary atresia. Pediatr Surg Int 2002;18(08):737-740

7 Lee H, Hirose S, Bratton B, Farmer D. Initial experience with complex laparoscopic biliary surgery in children: biliary atresia and choledochal cyst. J Pediatr Surg 2004;39(06):804-807, discussion 804-807

8 Martinez-Ferro M, Esteves E, Laje P. Laparoscopic treatment of biliary atresia and choledochal cyst. Semin Pediatr Surg 2005;14 (04):206-215

9 Ure BM, Kuebler JF, Schukfeh N, Engelmann C, Dingemann J, Petersen C. Survival with the native liver after laparoscopic versus conventional Kasai portoenterostomy in infants with biliary atresia: a prospective trial. Ann Surg 2011;253(04):826-830

10 Nakamura H, Murase N, Koga H, et al. Classification of biliary atresia in the laparoscopic era. Pediatr Surg Int 2016;32(12): 1209-1212

11 Ohigashi S, Taketa T, Shimada G, Kubota K, Sunagawa H, Kishida A Fruitful first experience with an $8 \mathrm{~K}$ ultra-high-definition endoscope for laparoscopic colorectal surgery. Asian J Endosc Surg 2019;12(03):362-365 